\title{
Cancer Genome Characterization Center
}

National Cancer Institute

\section{Source}

National Cancer Institute. Cancer Genome Characterization Center. NCI Thesaurus. Code C82428.

A multidisciplinary facility using various genomic and/or epigenomic analysis technologies for the systematic, high-resolution, comprehensive characterization of cancer-related genomic alterations in biomolecules obtained from highly-annotated, quality-controlled human cancer biospecimens. 